\title{
Can a Leaflet with Brief Verbal Instruction Teach Nepali Women How to Correctly Contract Their Pelvic Floor Muscles?
}

\author{
Delena-Mae Caagbay, ${ }^{1}$ Kirsten Black, ${ }^{1}$ Ganesh Dangal, ${ }^{2}$ Camille Raynes-Greenow ${ }^{3}$ \\ ${ }^{1}$ Discipline of Obstetrics, Gynaecology and Neonatology, University of Sydney, ${ }^{2}$ Department of Obstetrics \\ and Gynaecology, Kathmandu Model Hospital,Kathmandu, Nepal ${ }^{3}$ Sydney School of Public Health, University of \\ Sydney, Australia.
}

\section{ABSTRACT}

Background: Pelvic organ prolapse is a common disorder for women in Nepal causing symptoms and reduced quality
of life. Pelvic floor muscle exercise is a conservative treatment option for pelvic organ prolapse but the effective way
to teach women in Nepal is not known. The objective of this pilot study was to determine if an illustrative leaflet with
brief verbal instruction could teach Nepali women to correctly contract their pelvic floor muscles.
Methods: Fifteen parous women attending two outpatient gynecology clinics in Kathmandu Valley were interviewed
to assess their knowledge of pelvic organ prolapse and pelvic floor muscles exercise. Following verbal instruction and
an illustrative leaflet on how to contract their pelvic floor muscles, the transabdominal real time ultrasound was applied
to assess the muscle contraction.
Results: The median age of 15 participants was 45 years (range $18-75$ years) and 10 women had pelvic organ prolapse.
Some of the participants ( $9 / 15$ ) knew about pelvic organ prolapse but none were aware of the pelvic floor muscles.
After being taught how to contract their pelvic floor muscle, only 4 of 14 correctly contracted the pelvic floor muscle.
Conclusions: This study highlighted the low knowledge of the pelvic floor muscle, and brief verbal instruction with
an illustrative leaflet is also not sufficient in teaching Nepali women how to correctly contract their pelvic floor muscle.
Further research is needed to determine how to teach a correct pelvic floor muscle contraction for women with low
literacy in resource poor settings.

\section{INTRODUCTION}

Pelvic organ prolapse (POP) is a common condition for women globally and is one of the most widespread reproductive health problems in Nepal. ${ }^{1,2}$ POP occurs when the pelvic floor muscles (PFM) and supporting tissues are stretched, weakened or damaged allowing the bladder, uterus or bowel to descend into the vagina. ${ }^{3}$

Women who have weak or damaged PFMs have 4-11 times the risk of developing a POP when compared to women with good PFM function. ${ }^{4}$ Strengthening the PFMs is the first line conservative treatment option for POP and can improve symptoms, quality of life (QOL) and severity. ${ }^{5}$ However, teaching women to correctly exercise their PFMs is challenging and up to $50 \%$ may still do it incorrectly even after verbal instruction. ${ }^{6}$

The aim of this pilot study was to investigate whether brief verbal instruction in conjunction with an illustrated leaflet resulted in Nepali women being able to correctly contract their PFMs.

\section{METHODS}

In February 2016, a pilot study of parous women aged 18 years and above was conducted. The women were attending two gynecology outpatient clinics run by the Public Health Concern Trust: Kathmandu Model Hospital and Kirtipur Hospital, Nepal.

Women attending the hospital outpatient clinics were invited to participate in the study by their doctor. Initially, the doctor performed the Pelvic Organ Prolapse Quantification scale (POP-Q) to assess for POP. Following their clinical examination, a demographic questionnaire containing questions about their age, educational 
status, literacy, occupation and obstetric history was completed.

The face-to-face semi-structured interviews, which included questions regarding the participants' knowledge of POP and PFMs were conducted and recorded. The participants were asked to describe a POP, the causes and treatment options and how they learnt about the condition. They were also asked of their awareness of PFMs, their function and how to exercise them.

Participants were shown an illustrative leaflet with simple drawings demonstrating how to contract the PFMs correctly without breath holding or co-contracting other muscles. They were asked if they understood the images and if they could describe what was being illustrated. The participants were also encouraged to give feedback on the leaflet. The illustrative leaflet was developed in consultation with Nepali health professionals and public health workers who specialize in women's health.

Following this, the participants were given a detailed explanation of how to correctly contract the PFMs using the illustrative leaflet and verbal instruction. To assess their ability to contract the PFMs, 2D transabdominal real time ultrasound (RTUS) was performed to visualize the movement of the bladder as described in a previous study by Sherburn et al. ${ }^{7}$ The participants were instructed to "imagine stopping the flow of urine", "imagine trying to stop passing gas", and "squeeze and lift the muscles inside the pelvis". The RTUS findings were documented if the PFMs elevated, depressed or did not change and recorded how many millimeters of movement was detected.

The recorded interviews were transcribed verbatim into Nepali and translated into English; then reviewed by the interviewer to ensure the documentation was accurate. Due to the small sample size and homogeneity of responses, the results were collated and analyzed using a spreadsheet and descriptive statistics.

Ethics approval was granted through Institutional Review Committee of Public Health Concern Trust Nepal reference number 15027.

\section{RESULTS}

Fifteen parous women were recruited for this study with a median age of 45 years (range 18-75 years). Half of the participants had no formal education, five were illiterate and most $(11 / 15)$ engaged in unpaid work in their home and farm (Table 1). Out of the 15 participants, 10 had been diagnosed with a POP from the POP-Q examination performed by the gynecologist. Half the participants $(5 / 10)$ with a POP noticed their symptoms after their first or second delivery while the other half noticed after the third or more delivery. The most common reported symptoms of POP were 'bulging or something falling out between the legs' $(8 / 10)$ and 'pressure in the lower stomach or pelvis' $(7 / 10)$ (Table 2).

\begin{tabular}{lrr}
$\begin{array}{l}\text { Table 1. Participant demographic } \\
\text { (n=15). }\end{array}$ & information \\
Participant Demographics & 7 & Range \\
\hline Age & 8 & $18-40$ \\
& & \\
\hline & 10 & $\leq 41$ \\
\hline Age when married & 5 & $\geq 19$ \\
& & \\
\hline & 7 & $15-19$ \\
\hline Age of first pregnancy & 4 & $20-24$ \\
& 4 & $\geq 25$ \\
\hline & & 1 \\
\hline Number of deliveries & 3 & $2-3$ \\
& 7 & $\geq 4$
\end{tabular}

Highest level of education completed

\begin{tabular}{|ll|}
\hline None & 7 \\
\hline Primary & 3 \\
\hline High & 2 \\
\hline College & 3 \\
\hline
\end{tabular}

Occupation

$\begin{array}{lr}\text { Farmer/ } & 11 \\ \text { Housewife } & \\ \text { Service } & 4\end{array}$

Table 2. Pelvic organ prolapse questions for women who have a POP $(n=10)$.

\begin{tabular}{|c|c|c|}
\hline \multicolumn{3}{|l|}{ POP characteristics } \\
\hline POP Stage & & \\
\hline Stage I & 5 & \\
\hline Stage II & 1 & \\
\hline Stage III & 3 & \\
\hline & 1 & \\
\hline Stage IV & 4 & $18-30$ \\
\hline $\begin{array}{l}\text { Age of first noticing POP } \\
\text { symptoms }\end{array}$ & 6 & $\geq 31$ \\
\hline $\begin{array}{l}\text { Years of having a POP } \\
\text { symptoms }\end{array}$ & 6 & $0-10$ \\
\hline
\end{tabular}


We found very low knowledge of the PFMs, as none of the women recognized the term 'pelvic floor muscle'. When shown the illustrative leaflet with PFM exercises only one participant could explain what the images were illustrating. This participant responded, "the muscles are down and you have to tighten them". The participants were asked if there was anything confusing in the leaflet and they all responded that "everything" was confusing and a few suggested adding simple words and symbols to make it easier to understand. All the participants felt a leaflet would be useful and 8 of 15 suggested additional verbal instruction would be needed to explain the information.

Most of the participants had heard about POP (9/15) and responded with descriptions such as 'a fallen womb', 'muscles coming down' and 'something stuck down there'. The most common response to what causes a POP (9 respondents) was carrying heavy loads (6/9), childbirth (3/9) and work (3/9). When asked an openended question about POP treatment options, women responded with operation $(7 / 10)$ or a ring $(6 / 10)$; however, no participant suggested PFM exercises.

Fourteen participants had RTUS (one participant had a urinary catheter and RTUS was not performed as a full bladder is needed to observe movement), and only four could correctly elevate their PFMs while 10 incorrectly depressed the muscles and one had no change in movement. The description "imagine trying to stop the flow of urine" was the most effective description that resulted in the four participants correctly contracting the PFM. The verbal instruction "imagine trying to stop passing gas" resulted in three participants correctly elevating the PFMs and "now try squeezing and lifting the muscles inside your pelvis" assisted only one participant to perform a correct contraction. Common errors observed with the PFM contraction were participants holding their breath, bearing down and co-contracting their abdominal and leg muscles.

Of the four participants who correctly contracted their PFMs, two had never attended school, one completed four years of education and one had completed 10 years. These four participants were aged 38, 40, 42 and 64 three had a POP and two had given birth in hospital. There was no relationship between age, literacy, education, birthing history and pelvic floor disorders on the ability to correctly contract their PFMs.

\section{DISCUSSION}

Instructing women how to contract their PFMs was challenging as some may not understand and incorrectly contract the muscles even following education. In this study most $(10 / 14)$ of the participants incorrectly depressed or had no observed movement in their PFM contraction after receiving verbal and illustrative instruction. In contrast to these results, a recent study from the United States showed that $85.5 \%$ of women with a POP were able to correctly contract their PFMs after a brief verbal cue as assessed via digital vaginal palpation. ${ }^{8}$ The authors suggested that population-based prevention interventions, such as PFM exercise education, are worthwhile even without individual clinical assessment. Although caution should be taken when considering this study, as the participants were predominantly Caucasian and highly educated and may not be generalizable to women in resource poor settings.

Women in rural regions can face barriers in accessing individualized assessment and education from health professionals about POP and PFM exercise. ${ }^{9}$ Many of the studies demonstrating the positive effects of PFM exercise on POP required the participants to have multiple follow-up sessions with a health professional, which may not be feasible for many women who have accessibility issues. ${ }^{5,10}$ In remote regions of Nepal, health education is delivered predominantly through radio programs or female community health volunteers (FCHV). ${ }^{11}$ The FCHVs have basic health training and facilitate mother's groups using verbal instruction to deliver information on various maternal and child health topics. ${ }^{11}$ There is currently no data evaluating the most effective way that community health workers can teach PFM exercise to women living in rural regions.

Since we wanted to assess if verbal and illustrative instruction alone was sufficient in teaching women to correctly contract their PFMs, we opted to do the most minimally invasive assessment technique. For this reason, we used transabdominal RTUS to observe the correct 'lifting' action of PFM contraction rather than assess for muscle strength and defects. Transabdominal ultrasound has been found to be as reliable as transperineal ultrasound and also correlates with digital vaginal palpation in detecting the correct movement. ${ }^{12}$ Performing RTUS transabdominally reduced any direct sensory feedback to the PFMs that transperineal RTUS or digital palpation would have introduced.

None of the women in our study were familiar with the term 'pelvic floor muscles' and they had no comprehension of the PFM illustrations. Low knowledge of the PFMs is common for women globally and is associated with a higher risk of developing pelvic floor dysfunction. ${ }^{13}$ In Thailand, a study of 214 women attending a gynecology outpatient clinic showed that $50 \%$ of the respondents 
were unaware of PFM exercise. ${ }^{14}$ Low knowledge is also a problem in developed countries as seen in a Canadian study where women scored $40 \%$ on the Prolapse and Incontinence Knowledge Quiz. ${ }^{13}$ Increasing knowledge of the role of the PFMs is essential in motivating women to participate in ongoing self-management strategies. ${ }^{15}$

Another problem with having low knowledge of pelvic floor disorders and treatment options is that it reduces healthcare seeking behaviour. ${ }^{15}$ In our study the women reported having POP symptoms for an average of 16 years before seeking help, which is similar to a study conducted in Ethiopia where women suffered with a symptomatic POP for more than 10 years. ${ }^{16}$ Women in developing countries may not seek help due to feeling embarrassed, scared and accepting a POP as normal after giving birth. ${ }^{16-18}$ Increasing awareness and knowledge of pelvic floor disorders can help reduce stigma and increase healthcare seeking behaviour. ${ }^{19}$ Empowering FCHVs and other maternal health workers in Nepal to discuss PFM exercise with women and provide followup care and advice may help reduce and prevent the burden of POP. ${ }^{18}$

We acknowledge that this was a small pilot study, using a convenience sample of women attending a hospital clinic. However, we think the findings are important and we hypothesize that a larger study would have resulted in similar results. Even though the participants were within travelling distance to urban hospitals in Kathmandu Valley, it is likely that women living in remote regions of Nepal would have even less awareness of PFM exercise as a treatment option for POP. The small sample size provided adequate data for the qualitative interviews but the results of the ultrasound should be interpreted with caution. The results of this preliminary study will assist in adapting and creating a final version of the illustrative leaflet to be validated in future research. Further studies are needed with larger sample sizes to determine the most effective way to teach PFM exercise to Nepali women, especially those who live in remote regions who don't have access to health professionals for individualized assessment and instruction.

\section{CONCLUSIONS}

This study highlighted the inadequacies of brief verbal education and an illustrative leaflet in teaching Nepali women to correctly contract their PFMs. Developing strategies and resources to assist community health workers in teaching women PFM exercise is important in achieving long-term prevention and management of POP in Nepal and other similar resource poor settings.

\section{REFERENCES}

1. Thakar R, Stanton S. Management of genital prolapse. BMJ. 2002 May 25;324(7348):1258-62. [Link]

2. Bodner-Adler B, Shrivastava C, Bodner K. Risk factors for uterine prolapse in Nepal. Int Urogynecol J. 2007 Nov 1;18(11):1343-6.[Link]

3. Shek KL, Dietz HP. Assessment of pelvic organ prolapse: a review. Ultrasound Obstet Gynecol. 2016;48(6):681-92. [Link]

4. Braekken IH, Majida M, Ellstrom Engh M, Holme IM, Bo K. Pelvic floor function is independently associated with pelvic organ prolapse. BJOG. 2009;116(13):1706-14. [Link]

5. Li C, Gong Y, Wang B. The efficacy of pelvic floor muscle training for pelvic organ prolapse: a systematic review and meta-analysis. Int Urogynecology J. 2016;27(7):981-92. [Link]

6. Bump RC, Hurt WG, Fantl JA, Wyman JF. Assessment of kegel pelvic muscle exercise performance after brief verbal instruction. Am J Obstet Gynecol. 1991;165(2):322-9. [Link]

7. Sherburn M, Murphy CA, Carroll S, Allen TJ, Galea MP. Investigation of transabdominal real-time ultrasound to visualise the muscles of the pelvic floor. Aust J Physiother. 2005;51(3):167-70.[Link]

8. Henderson JW, Wang S, Egger MJ, Masters M, Nygaard I. Can women correctly contract their pelvic floor muscles without formal instruction? Female Pelvic Med. 2013;19(1):8-12.

9. Rizk DE. Minimizing the risk of childbirth-induced pelvic floor dysfunctions in the developing world: "preventive" urogynecology. Int Urogynecol J Pelvic Floor Dysfunct. 2009;20(6):615-7.[Link]

10. Byrne A, Hodge A, Jimenez-Soto E, Morgan A. What works? Strategies to increase reproductive, maternal and child health in difficult to access mountainous locations: a systematic literature review. PLoS ONE [Electronic Resource]. 2014;9(2):e87683.[Link]

11. Byrne A, Hodge A, Jimenez-Soto E, Morgan A. Looking beyond supply: a systematic literature review of demandside barriers to health service utilization in the mountains of Nepal. Asia Pac J Public Health. 2013;25(6):438-51. [Link]

12. Thompson JA, O'Sullivan PB, Briffa NK, Neumann 
P. Comparison of transperineal and transabdominal ultrasound in the assessment of voluntary pelvic floor muscle contractions and functional manoeuvres in continent and incontinent women. Int Urogynecol J. 2007;18(7):779-86.[Link]

13. Berzuk K, Shay B. Effect of increasing awareness of pelvic floor muscle function on pelvic floor dysfunction: a randomized controlled trial. Int Urogynecol J. 2015;26(6):837-44.[Link]

14. Tantisiriwat N, Manchana T. Knowledge and acceptance of Thai women toward the pelvic floor muscle training. J Medi Assoc of Thailand= Chotmaihet Thangphaet. 2014;97(1):7-11.[Link]

15. Tannenbaum C, Drali R, Holroyd-Leduc J, Richard L. Lessons Learned: Impact of a Continence Promotion Activity for Older Community-Dwelling Women. Neurourol Urodyn. 2010;29(4):540-4.[Link]

16. Gjerde JL, Rortveit G, Muleta M, Adefris M, Blystad A. Living with pelvic organ prolapse: voices of women from
Amhara region, Ethiopia. Int Urogynecol J. 2016;1-6[Link]

17. Walker GJA, Gunasekera P. Pelvic organ prolapse and incontinence in developing countries: review of prevalence and risk factors. Int Urogynecol J. 2011;22(2):127-35. [Link]

18. Pradhan S. Unheeded Agonies - A study on uterine prolapse prevalence and it's causes in Siraha and Saptaria districts. Kathmandu, Nepal: Women's Reproductive Rights Program (WRRP). 2007.

19. Shrestha B, Onta S, Choulagai B, et al. Women's experiences and health care-seeking practices in relation to uterine prolapse in a hill district of Nepal. BMC Women's Health. 2014;14(1):20.[Link] 